\title{
Handbook of Research on Computational Arts and Creative Informatics
}

James Braman

Towson University, USA

Giovanni Vincenti

Gruppo Vincenti, S.r.I., Italy

Goran Trajkovski

Laureate Higher Education Group, USA

Information Science REFERENCE
INFORMATION SCIENCE REFERENCE

Hershey • New York 


\begin{tabular}{|c|c|}
\hline Director of Editorial Cont & linger \\
\hline Senior Managing Editor: & Jamie Snavely \\
\hline Managing Editor: & Jeff Ash \\
\hline Assistant Managing Editor: & Carole Coulson \\
\hline Typesetter: & Chris Hrobak \\
\hline Cover Design: & Lisa Tosheff \\
\hline Printed at: & Yurchak Printing Inc. \\
\hline $\begin{array}{l}\text { Published in the United States } \\
\text { Information Science } \\
701 \text { E. Chocolate A } \\
\text { Hershey PA 17033 } \\
\text { Tel: 717-533-8845 } \\
\text { Fax: 717-533-8661 } \\
\text { E-mail: cust@igi-gl } \\
\text { Web site: http://ww }\end{array}$ & $\begin{array}{l}\text { America by } \\
\text { Reference (an imprint of IGI Global) } \\
\text { nue }\end{array}$ \\
\hline $\begin{array}{l}\text { and in the United Kingdom by } \\
\text { Information Science } \\
3 \text { Henrietta Street } \\
\text { Covent Garden } \\
\text { London WC2E 8LL } \\
\text { Tel: } 44207240085 \\
\text { Fax: } 4420737906 \\
\text { Web site: http://ww }\end{array}$ & Reference (an imprint of IGI Global) \\
\hline
\end{tabular}

Copyright (C) 2009 by IGI Global. All rights reserved. No part of this publication may be reproduced, stored or distributed in any form or by any means, electronic or mechanical, including photocopying, without written permission from the publisher.

Product or company names used in this set are for identification purposes only. Inclusion of the names of the products or companies does not indicate a claim of ownership by IGI Global of the trademark or registered trademark.

Library of Congress Cataloging-in-Publication Data

Handbook of research on computational arts and creative informatics / James Braman, Giovanni Vincenti and Goran Trajkovski, editors.

p. cm.

Includes bibliographical references and index.

Summary: "This book looks at the combination of art, creativity and expression through the use and combination of computer science, and how technology can be used creatively for self expression using different approaches"--Provided by publisher. ISBN 978-1-60566-352-4

(hardcover) 1. Technology and the arts. 2. Creation (Literary, artistic, etc.) 3. Computer science. I. Braman, James, 1981- II. Vincenti,

Giovanni, 1978- III. Trajkovski, Goran, 1972- NX180.T4H36 2009

$700.285--d c 22$

\section{7}

British Cataloguing in Publication Data

A Cataloguing in Publication record for this book is available from the British Library.

All work contributed to this book is new, previously-unpublished material. The views expressed in this book are those of the authors, but not necessarily of the publisher. 


\title{
Chapter XIII Memory Association Machine
}

\author{
Benjamin David Robert Bogart \\ Simon Fraser University, Canada
}

\begin{abstract}
"Memory Association Machine" (also known as "Self-Other Organizing Structure \#1") is the first prototype in a series of site-specific responsive installations inspired by cognitive processes. The artist provides a mechanism that allows the structure of the artwork to change in response to continuous stimulus from its context. Context is defined as those parameters of the environment that are perceivable by the system and make its place in space and time unique. "Memory Association Machine" relates itself to its context using three primary processes: perception, the integration of sensor data into a field of experience, and the free-association through that field. "Memory Association Machine" perceives through a video camera, integrates using a Kohonen Self-Organizing Map, and free-associates through an implementation of Liane M. Gabora's model of memory and creativity.
\end{abstract}

\section{INTRODUCTION}

This text describes and frames the first prototype in a body of work that aims to create artworks that find their own relationship to their context. These artifacts are embodied, meaning that they are manifested in a physical ${ }^{1}$ form and are effected by, and effect, the world around them. The artifacts could be considered creative machines in that they transform material from their context into an original representation. The machine creates this representation through a mechanism provided by the artist. My research aims to use artistic enquiry to develop a metapractise that binds the practises of responsive electronic media art, site-specific art, and artificial intelligence. This meta-practise includes theory from the philosophy of embodiment and is developed through the creation of embodied artifacts-as-processes - artifacts composed of computational processes. The material of the artwork is a set of computational processes that are causally connected to the physical world. The mechanism of the artwork is intended to exhibit emergent properties through the negotiation between software and physical context. 
The system's processes are causally connected to the outside world through sensors (inputs) and external properties (outputs). How can an artifact-even a process - find a relationship to its context? Artifacts such as "Memory Association Machine" form an embodied relationship with their context in two ways; firstly, by being embodied so that they can both change and be changed by their context, and secondly, by having their structure altered through the process of embodiment. A more detailed account of the creation of "Memory Association Machine" is available in "Memory Association Machine: An Account of the Realization and Interpretation of an Autonomous Responsive Site-Specific Artwork" (Bogart, 2008). The process of embodiment is the negotiation between the subject and the objectthe relationship between seeing and acting.

This text begins by weaving a theory of practise which has been, and is still being, developed. The practise is focused on the fundamental relationship between the artwork (artifact-as-process), the artist (author), and the world in which they are both embodied. A description of "Memory Association Machine" is interjected throughout the framing and followed by a detailed descrip- tion of the system's architecture. Future work is presented at the conclusion of the text.

\section{The Artifact}

The formalization of the creative process is made up of two iterative sub-processes: realization and interpretation. The relationship between these processes is pictured in Figure 1. Realization is the path of intention from the artist to the world, whereas interpretation is the path of causation from the world back to the artist. This formalization is influenced by a theory of embodiment, the "intertwining", that binds the mind and body according to the phenomenology of Merleau-Ponty (1968). For each pair of actions (realization and interpretation) a frame ${ }^{2}$ is created which colours the artifact (at that moment in time) with the tension between the expected results of realization and the following interpretation. Realization occurs when the artist chooses to effect the world in some way that manifests physically-for example, choosing the colour yellow for a particular region of a painting.

Interpretation is when the artist observes, experiences, and attributes value to the results

Figure 1. The artist realizes the artifact, which is then interpreted

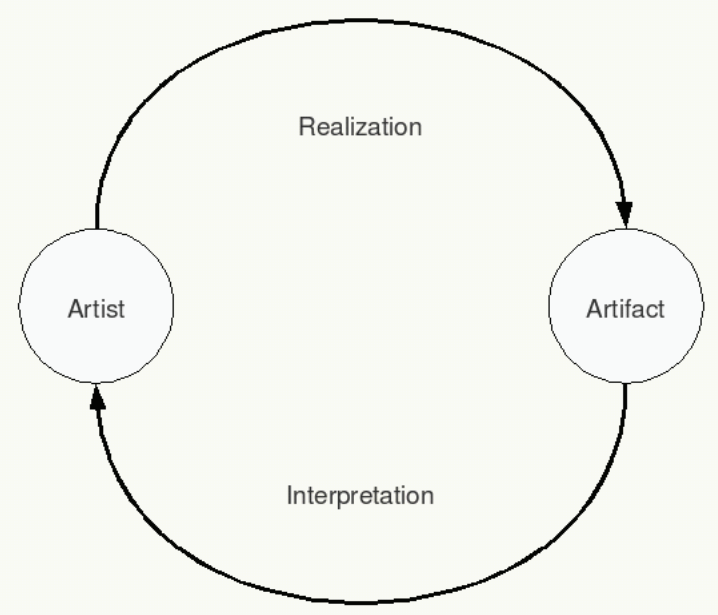


of the realization-for example, seeing yellow in a particular spot on a canvas. The perception of yellow results in the inevitable associations resulting from that stimulus, which have value in terms of the artist's experience. The painter may decide that the tone of yellow is not quite what he or she intended and adds more white to the paint. The artifact is transformed through the iterative dialectic between realization and interpretation. Future choices are made, in light of not just the current frame, but in the context of all past frames. ${ }^{3}$
Both the artifact and the artist's intentions are transformed through this process. The artist may interpret the artifact differently than the viewer. Artifacts-as-processes, on the other hand, are both snapshots of their own creation and enacted processes in themselves. Embodied artifacts-asprocesses are machines that are constantly changing their own properties to form a relationship to, through the reflection and interpretation of, their embodied context.

Figure 1 is a simplified representation. It is not uncommon for a single artifact to contain

Figure 2. The nested realization-interpretation loop

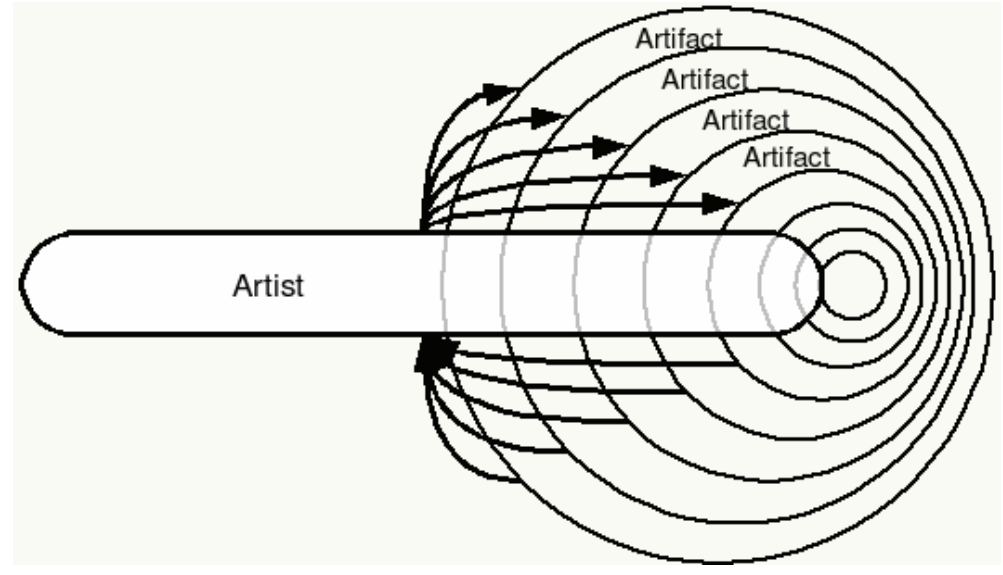

Figure 3. The realization-interpretation loop of the embodied artifact-as-process

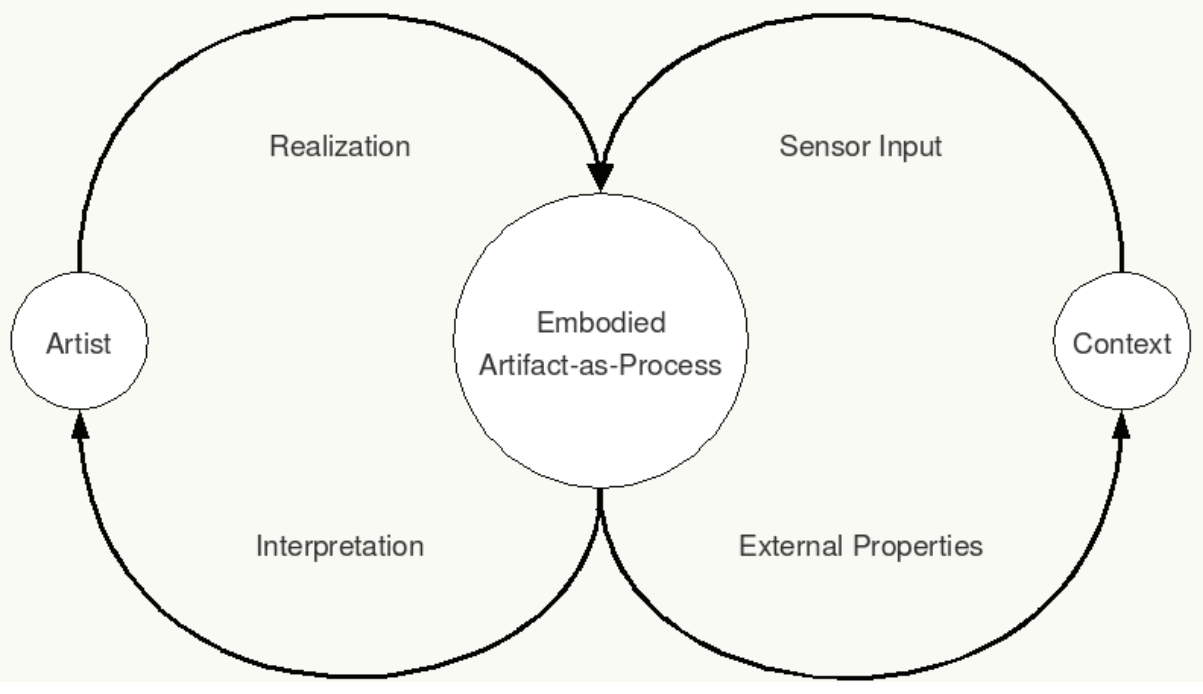


other artifacts, which in turn contain additional components, as pictured in Figure 2, where each artifact contains another artifact. ${ }^{4}$ At each level in the nested structure there is a different realizationinterpretation frame. The method of realization and criteria of interpretation may be different at each level in the structure. The time it takes to complete a single loop is entirely dependent on the nature of the creation. Some loops proceed very quickly - for example, the choice of a colour and the placement of a single blob of pigment on the canvas. Others take much more time-for example, the creation of a skyscraper.

\section{Embodiment}

"Memory Association Machine" is an embodied artifact-as-process. The embodied artifact-asprocess is made up of two primary components: the software and the context. In the creation of embodied artifacts-as-processes the artist's intentions are often encoded in software (instructions for a computational process). As the software artifact is the primary medium in which I work, I consider software as my artistic material. The software material is a set of computational relations between symbols (variables). It is not a process but a static text that encodes a set of instructions. Software can become an embodied artifact-as-process when a subset of its constituent symbols become inputs and outputs that are causally connected to the physical world and perceivable by the viewer. It is this causal relationship between the world and the software that activates its instructions. The material of the work shifts from the representation of a process to action in the physical world. ${ }^{5}$ The behaviour (the change of external properties) of the embodied artifactas-process is a collaboration between the artist's intention, as encoded in software, and the context in which the artifact-as-process is embodied. Figure 3 depicts the realization-interpretation loop of an embodied artifact-as-process, where arrows represent causal relationships.
In contemporary artistic practise it is often the text accompanying the work that is expected to communicate the work's concept and purpose. This text is an artifact that exists independently from the artwork. Through realization and interpretation, the text, the software, and the artist's intention reach an equilibrium. What the machine means and what the machine does are unified. The software is the mechanism-but the process, executed in context, is the artwork. The result is a collaboration between the artist and the context. The role of the context must be embraced. Once the embodied system's behaviour has been interpreted, the software can be altered in order to more finely unify the artist's intention and the system's behaviour. The artist is then working in two different materials - the material of the software, which is impacted upon by the artist, and the material of the embodied process, which impactsitselfon the artist. The interaction between the software and the physical world is not totally deterministic. The artist must relinquish control and allow the process to be driven equally by the software and the context. In "Memory Association Machine," the driving intention is that the artifact-as-process acts beyond of the intentions of the artist.

\section{Artistic Enquiry}

Historically, the artist is "imagined as an isolated figure of exceptional creative powers who suffers for his art." (Barker et al., 1999). There is a mythology surrounding the "creative genius" and the artifacts that the, predominantly male, artist creates. The artifact is then a record of genius which is often collected and fetishized. The artifact is manifested in the physical world. It becomes meaningful as its references are processed through the experience of the viewer. Why should the artist have more authority to attribute meaning to an artifact than the viewer?

Some approaches to art practise reject this notion of artwork as an expression of genius, 
and break the mythos of creativity, by shifting the emphasis away from the artifact toward the process itself(Possiant, 2007). ${ }^{6}$ What is produced when the purpose of the work is not the creation of an art object, but an exploration of the very creative process itself? There are two products of this enquiry. The first is the artifact-as-process itself. The second is the knowledge that results from the artistic enquiry. This knowledge is manifested in the artifact-as-process and around it through documentation, rhetoric, sharing, and discussion.

This artistic enquiry is centred on the artistic practises of responsive electronic media and site-specificity. For a survey of electronic media art see "Information Arts" (Wilson, 2002). The discipline of site-specific artwork aims to create work that gives "....itself up to its environmental context, being formally determined or directed by it" (Kwon, 2004). Kwon (2004) and Kaye (2000) provide a background on site-specific practises. This body of work explores the qualities of embodied creativity through the artistic practise of developing artifacts-as-processes that find their own relationship to their context.

\section{GROWING FORM FROM CONTEXT}

In order to have "Memory Association Machine" find its own relationship to its context in a way that is not externally predetermined nor random, it is natural to look to cognitive science. The most relevant application of cognitive science happens in the discipline of artificial intelligence, which seeks to create software and hardware that exhibits some of the properties of human beings. In order for "Memory Association Machine" to relate to its context, the use of unsupervised connectionist artificial intelligence approaches is appropriate, as the behaviour of the system should not be dependent on an external knowledge-base provided to it. Since "Memory Association Machine" is an embodied system, the physical environment becomes the "training" data for its artificial intelligence.

\section{Methodology of Artistic Enquiry}

As this research project is contextualized as an artistic enquiry, it is important to describe how the creation of these artworks is approached. The software is built piece-by-piece while the system is connected to its context. As the artwork is considered the result of the machine's negotiation with its context, the artwork can only be appropriately interpreted in its embodied context. The system's components are initially developed in isolation and attached to the rest of the system as early as possible. This method of building the system incrementally, and testing it in an embodied context at each step of development, resembles the methodology discussed in "[i]ntelligence without representation" (Brooks, 1992). The software development occurs in two modes. The first mode is an intuitive approach ${ }^{7}$ that serves to get the basics of the system up and running - with arbitrary choices, random variables, and placeholders - so that the system can be evaluated in context, rather than in isolation. The second mode of grounded refinement involves returning to the results produced in the first mode and removing arbitrary choices by situating them in theory. For "Memory Association Machine," the source of theory comes from three main sources: connectionist artificial intelligence, specifically Kohonen "Self-Organizing Maps"; theories of creativity, specifically those present in the "Cognitive Mechanisms Underlying the Creative Process" (Gabora, 2002); and the theory that is created through the meta-practise itself. Arbitrary and random variables are either replaced with variables that refer to the embodied context, or with values consistent with available theory. The software development happens in the Pure Data visual programming system (Puckette etal., 1996), where each step of development is managed by the subversion version control system(CollabNet, 
2000). Through each development iteration, the system is evaluated against various criteria. The most important criteria is my subjective interpretation of the system's embodied behaviour. The system is observed in two modes. The first mode entails direct observation of the system's external properties in context. This mode of observation tends towards a qualitative approach. The system keeps a detailed log of key variables, as it negotiates with its context, which is examined in the second mode using R(R Development Core Team, 2007). At regular intervals the system archives an image of its external properties. The archive and logs are used to expose software errors and track the system's process over time. Additionally, the logs show a record of the stability of the system as it is intended for long-term public exhibition. The artist also keeps a journal of the development process.

\section{Why Use Artificial Intelligence?}

In order to answer this question one must first define Artificial Intelligence (AI). A general definition states that AI is "part of computer science concerned with designing intelligent computer systems, that is, systems that exhibit the characteristics we associate with intelligence in human behavior-understanding language, learning, reasoning, solving problems and so on." (Barr and Feigenbaum, 1981). Although this definition does not directly mention creativity it is certainly an aspect of human intelligence. Boden (2004) argues that machines can be "considered" creative in the same way that machines can be thought of as intelligent according to the "Turing Test” (Turing, 2004). Stephen Wilson considers the relationship between AI and art:

Artificial Intelligence is one of these fields of inquiry that reaches beyond its technical boundaries. At its root it is an investigation into the nature of being human, the nature of intelligence, the limits of machines, and our limits as artifact ${ }^{8}$ makers. I felt that, in spite offalling in and out of public favor, it was one of the grand intellectual undertakings of our times and that the arts ought to address the questions, challenges, and opportunities it generated. (Wilson, 1995)

In this project I expect the system to be creative by it defining its own relationship to its context. Furthermore, I expect that the artwork makes creative choices that manifest themselves through the artwork's external properties, which refer to the context and represent the machine's unique integration of its experience. AI is the only discipline - with its roots in cognitive science - that explores those questions of creativity through the implementation of systems that embody aspects of the human mind. ${ }^{9}$ For this reason, AI is the first logical discipline to consider for technique. That being said the central basis of intelligence in AI is problem solving. This research project, with its basis in artistic enquiry, does not consider creativity as problem solving. For a critique of AI's focus on problem solving, see Agre (1997).

What techniques and processes from AI could allow an artwork to form its own relationship to context? To use non-AI software techniques, I, as the artist, would determine how the work relates to its context, rather than it finding its own connection. Those AI techniques, such as SelfOrganizing Maps (Kohonen, 2001), that allow the system to reorganize itself based on sensor input, are a likely requirement to build the mechanisms that allow a machine to relate itself to its context without using problem solving approaches.

\section{THE "MEMORY ASSOCIATION MACHINE" ARCHITECTURE}

"Memory Association Machine" has three states: waking, dreaming, and suspended; and is composed of two primary systems: the "Memory System" and the "Free-Association System". Both of these systems are networks of numerous identi- 
cal components, or units. The selection of state is currently based on a predetermined schedule. The system is initially in a waking state. In this state the system observes its context by pointing the camera in a random direction, collecting images, and selecting a new random direction each twelve seconds. For each direction in which the camera looks, a unit of the free-association system is activated. This results in a cascade of activations in the system's previous experience.

In the dreaming state the camera ceases to explore and images are not collected. A timeseeded random value activates a unit in the freeassociation system each twenty-five seconds. During the dreaming state, while the system is isolated from external stimulus, the random activations propagate through the free-association network. This propagation calls up elements from previous experience which are not initiated by the embodied context.

In the suspended state the displays are put into DPMS ${ }^{10}$ sleep and the dreaming state ends. This state exists to reduce wear on the camera and displays when there is both little light and activity in the machine's physical context.

The memory and free-association networks interact with the embodied context to generate the system's external properties. The memory system is the mechanism through which the system stores and integrates its previous experience. Central to the memory system is a Kohonen Self-Organizing Map (SOM) (Kohonen, 2001). The SOM is an unsupervised artificial neural network which acts as an arbitrary pattern classifier. The choice of which category a particular input is associated with is based on its similarity to other inputs. The patterns presented to the SOM in "Memory Association Machine" are the images captured by the camera. The result is a SOM which stores the machine's visual experience of its context and compares it with its remembered experience.

The free-association system is a network of simple units which is independent of the SOM. This network allows the propagation of signals between units - the basis of the free-association system - and is similar to a cellular automata. The activation of units in the network selects images from the system's memory. This free-association is considered a creative act, as the choices of what images to select are not predetermined, but a result of the behaviour of the entire system. This model of creativity is based on "[t]he cognitive mechanisms underlying the creative process" (Gabora, 2002).

The direction in which the camera looks is currently controlled by three time-seeded random

Figure 4. The system architecture of "Memory Association Machine"

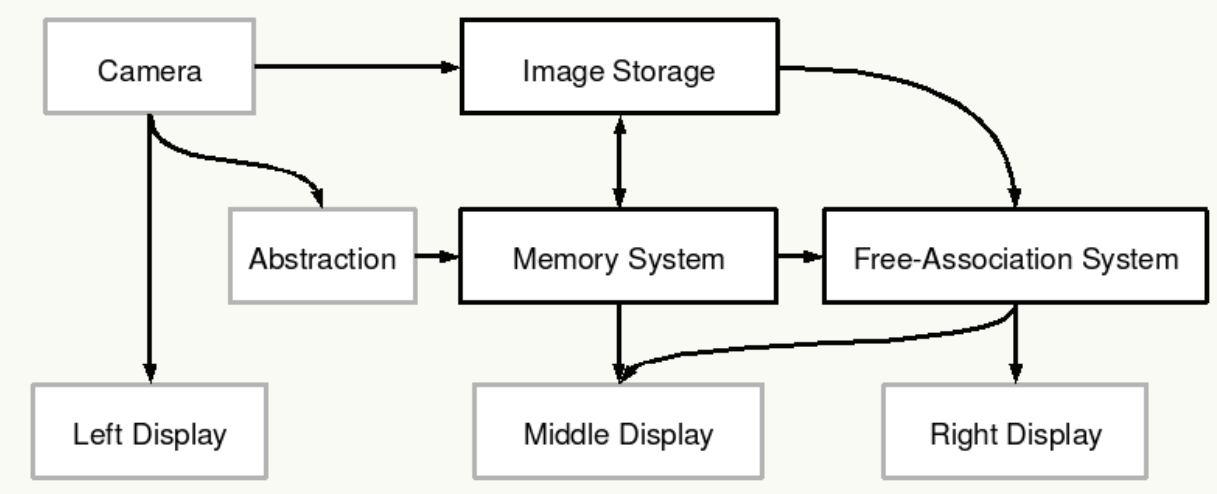


variables corresponding to the camera's pan, tilt, and zoom parameters. Figure 4 shows an overview of the "Memory Association Machine" architecture.

\section{Memory System}

Also known as a Kohonen network, a SelfOrganizing Map, is an unsupervised artificial neural network (ANN) designed expressly for the purpose of classification. An ANN is an AI approach inspired by neurophysiology. An unsupervised ANN does not depend on the "correct" answer, provided by the researcher, to "learn". Rather, these networks restructure themselves based on input patterns through the process of training. These systems are characterized by being composed of numerous simple components which are massively interconnected. For a survey of ANNs see Medler (1998). The SOM is a nonlinear projection of a high-dimensional data-space onto a low dimensional "feature map" that preserves topology. ${ }^{11}$ A SOM is able to categorize an arbitrary input pattern, with a finite number of dimensions, into a finite, and fixed, number of categories. ${ }^{12}$ The SOM is considered a "projection" as it maps values from the input space onto values in the output space. The points in the input space are the input patterns. The points in the output space are the categories.

The SOM consists of a network of units, ${ }^{13}$ each corresponding to a potential category of input. These units are usually arranged in a 2D Euclidean lattice which serves as the output space. Through training, each of the input patterns is associated with a particular category. The input space is defined as a set of real numbers, $\Re^{n}$, with $n$ dimensions. Points from the input space (the space of all possible inputs) are mapped to points in the output space (the position of all the units in the map). Points in input space are defined as $x=\left\{\xi^{1}, \xi^{2}, \ldots, \xi^{n}\right\} \in \Re^{n}$, where $x$ is a single point defined by the magnitudes of each dimension $(\xi)$ in the set of all possible inputs. The set of all input patterns is denoted as $x_{i}^{j}$, where $i$ is the number of input patterns, and $j$ is the number of dimensions, where $1 \leq j \leq n$. Similar inputs are associated with similar categories. Inputs are considered similar based on the sum of the distances between their vector components, $\operatorname{dist}\left(x_{1}, x_{2}\right)=\sum_{j=1}^{n}\left(x_{1}^{j}-x_{2}^{j}\right)^{2}$. The smaller the distance the more similar the inputs are. ${ }^{14}$ Categories are considered similar based on their Euclidean distance in output space. Dissimilar inputs can end up being associated with similar categories when two or more highly dissimilar clusters of points are presented to the network. The clusters then compete for space in the finite SOM - causing folds where the Euclidean distance between units, in output space, is not proportional to the distance between the patterns they are associated with.

Each unit of the network has a number of "sensors". Each sensor corresponds to one dimension of the input space. The units have as many sensors as the input space has dimensions. Each unit has a "code-book vector", also known as the weight vector, which contains the same number of elements as the unit has sensors. It is defined as $m_{i}=\left\{\mu_{i}^{1}, \mu_{i}^{2}, \ldots, \mu_{i}^{n}\right\}$, where $i$ is the index of the unit. Each unit has a "neighbourhood", denoted $N_{i}$, which is defined by the set of units within a fixed radius, ${ }^{15}$ in output space, of the unit $i$. During training the values of the code-book vectors approach the values of the input patterns. The SOM training process is an attempt to change the network's internal structure (its code-books) to more closely match the structure of the input patterns. The network attempts to mimic, using fewer dimensions, the structure of the patterns it is presented with. This is the "topology preserving" aspect of the SOM. The SOM training procedure, as used in "Memory Association Machine", is as follows:

1. Set the code-books of all units to $0 .{ }^{16}$ $m_{i}=0$ 
2. Present the network with an input pattern. (Give the units something to compare their code-books to.)

3. Select the unit that contains the codebook with the smallest sum of the square of the distances, in all dimensions, compared to the current input pattern. This is called the "Best Matching Unit" or BMU. (The BMU most closely resembles the most recent input pattern. $\left.{ }^{17}\right)$ $c=\operatorname{argmin}_{i}\left(\sum_{j=1}^{n}\left(x_{i}^{j}-m_{i}^{j}\right)^{2}\right)$, where $c$ is the index of the BMU.

4. Check if each unit is in the neighbourhood of the BMU.

5. ForeachunitintheneighbourhoodoftheBMU, add to the code-book vector the difference between the input pattern and the code-book multiplied by the learning rate, denoted $\alpha()$. $m_{i}(t+1)=m_{i}(t)+\alpha(t)\left(x(t)-m_{i}(t)\right)$, where $t$ is the current time step.

6. Repeat from 2.

In a canonical SOM, the amount that the codebooks are changed (learning rate), and the size of the neighbourhoods (neighbourhood function), both decrease monotonically over time. This is not the case in the "Memory Association Machine" implementation (to be discussed later). Training is complete when the mean of the difference between all input patterns and all units' code-books, $\operatorname{mean}_{i}\left(\sum_{j=1}^{n}\left(x_{i}^{j}-m_{i}^{j}\right)^{2}\right)$, ceases to decrease. The procedure typically takes from hundreds to thousands of iterations. ${ }^{18}$ As the code-book vectors are often randomized on initialization, in the canonical SOM, it is not known which inputs will be associated with which units at the start of training.

As the "Memory Association Machine" camera explores its context, the system creates a field of experience which is organized using the SOM. ${ }^{19}$ Seen in output space, the SOM attempts to organize its experience - in the form of visual images collected from its context-into a series of regions that contain similar experiences that are apart from areas of dissimilar experiences. The camera image is fed into the computer as a full-frame $30 \mathrm{fps}$ video stream. At twelve-second intervals, the 12x12 unit SOM is fed with a 100x75 pixel RGBA sub-sampled frame of the video stream. The raw values for each of those 7500 pixels, represented as four RGBA ${ }^{20}$ floating point values, correspond to the 30,000 sensors of each $\mathrm{SOM}$ unit. The SOM is used to index, rather than store, the images. The BMU, corresponding to a particularimage, is used to specify the index of the memory location where the image is stored. The storage area contains the same number of images as the SOM has units. If more than one image is associated with the same category, the most recent image replaces the previous. The mean time an image is held in the system is approximately two hours and a range of approximately thirty-three hours. ${ }^{21}$ As a single image is stored for each unit in the network, the memory for unusual images is longer than for common images. Common images are seen more often and are therefore replaced more often. Images are stored at their full resolution-not the sub-sampled resolution fed to the SOM.

"Memory Association Machine" represents the memory system as an Euclidean lattice, seeFigure 5. The visual representation of the system is created using the Pure Data "Graphics Environment for Multimedia" (GEM) (Mark Danks, 1995-). The display area is divided by the number of units. Each unit occupies the same amount of display space. The units are represented as circles with a Gaussian alpha channel. The "feathered" edge allows the memories, associated with neighbour units, to blend together. As a result, the structure within the images take precedence over the structure of the lattice. Only in regions associated with images that have very little spacial variation - for example, those that are out of focus-does the lattice of circles become visible. ${ }^{22}$

"Memory Association Machine" is intended to be in constant negotiation with its context. The SOM must be trained continuously to incorporate 
Figure 5. The representation of the memory system's field of experience

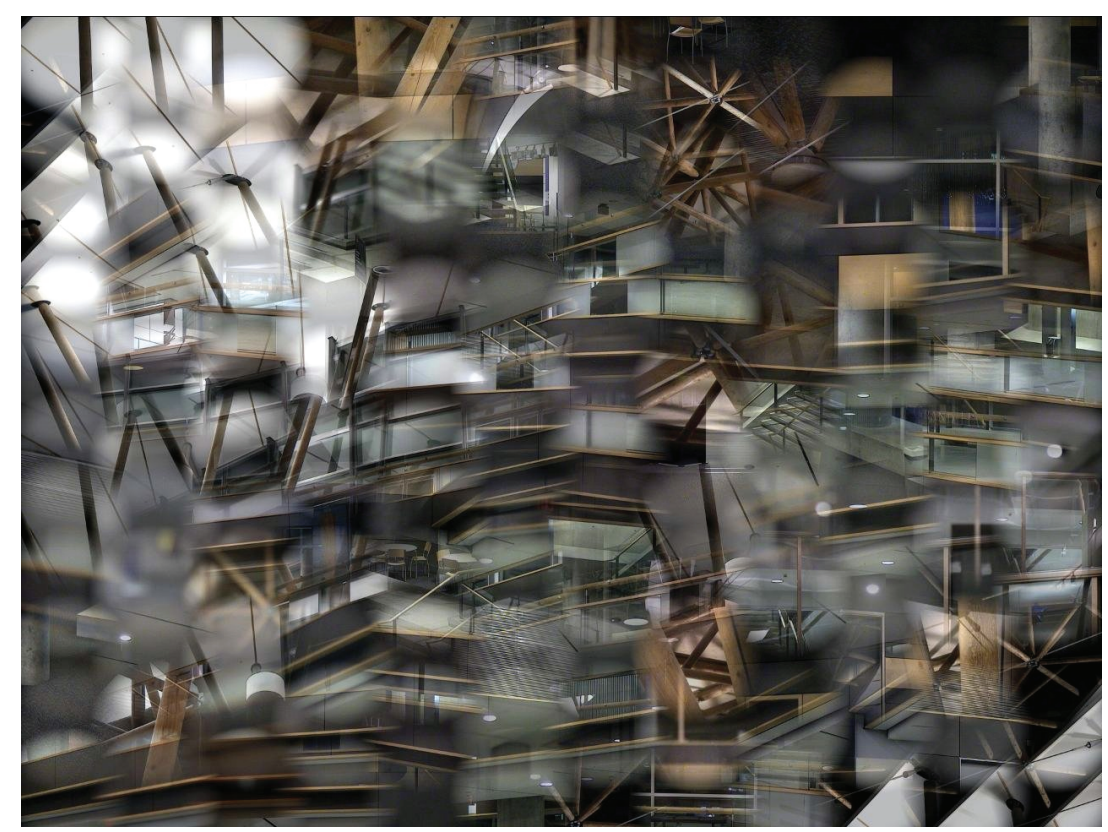

new experiences in its structure. A significant design difference between the canonical SOM and that implemented in "Memory Association Machine" is that the network is constantly converging, but is not meant to, and cannot, reach convergence. Allowing the SOM to converge implies that its process of relating to its context could be complete. As the context changes, so should the SOM structure. Continuous training is enabled by using cyclic learning and neighbourhood functions. These functions control the rate at which the self-organization evolves and is refined. They are driven by the cosine equation defined as $\frac{\cos (t / 100)+1}{2}$, where $t$ is the iteration counter that wraps from $628\left(» 200\right.$ ) to $0 . .^{23}$ The function is iterated each twelve seconds and results in a period of approximately two hours. The cyclical functions allow the SOM to respond to a continuous flow of new input patterns and integrate them into a constantly reorganizing field of memory. Code-books are being constantly refined through the training process. This makes the initial codebooks increasingly insignificant over time. ${ }^{24}$ As the training process is continuous, the SOM is replacing existing structure, created as a result of previous experience, with structure that reflects current experience. The network oscillates between durations of large and small change. ${ }^{25}$

The relatively small number of units in the SOM, combined with the high diversity of data in the system's embodied context, makes the structure of the memory representation highly complex. It is often interpreted by viewers of the installation as unorganized. The resolution of the image fed into the SOM was determined through trial and error based on the system's performance. The discrepancy between the resolution of the images used in the memory representation (320x240 pixels) and the resolution the SOM is presented with (100x75 pixels) likely compounds the problem. The memory representation takes on a quality very different than that of evenly distributed randomness. ${ }^{26}$

\section{Free-Association System}

Each time a new experience is perceived, it sets forth a stimulation within the content of memory. 
This stimulation calls up similar (nearby in the feature map) experiences from the past. These activations stimulate other experiences - traversing the memory from the similar to the dissimilar. ${ }^{27}$ As the traversal progresses, the energy in each stimulation decreases. Each subsequent experience is stimulated less than the previous one. As the free-association traces a branching path through the system's experience, the memories intersected by that path are visualized as a cinematic montage on the right display.

The model of stimulation and propagation is a custom network made up of Pure Data abstractions. ${ }^{28}$ When the camera looks in a direction, before the image is stored, the free-association unit associated with the BMU (the SOM unit that most resembles the image from the camera) is sent an activation signal. This initial activation is set at the maximum value of 1 . Once activated, each unit selects two random numbers between 0 and 7 -corresponding to the 8 directions in which a signal can be propagated. The signal is then transferred to its neighbours ${ }^{29}$ within that random range. Before it is propagated the signal is decreased by $20 \%{ }^{30}$ so that it falls off proportional to the distance between the initial activation and each receiving unit. Signals are propagated between free-association units using a custom message containing:

- $\quad$ The $(\mathrm{x}, \mathrm{y})$ position of the sending unit

- The $(\mathrm{x}, \mathrm{y})$ position of the destination unit

- The value of the signal.

The use of $(\mathrm{x}, \mathrm{y})$ pairs, rather than unique indices, was chosen to facilitate the calculation of which units are the immediate neighbours of the unit propagating the signal. Messages are sent to all units in the network, but only the units whose $(\mathrm{x}, \mathrm{y})$ indices match the destination processes the message.

There is a temporal delay in the propagation of signals. Each direction delays the signal for a time specified by a time-seeded random number rang- ing between 500 and 1000 milliseconds. This is done to reduce the number of activations occurring nearly simultaneously. In addition, when a unit is activated it becomes inhibited. For a duration of two seconds, that unit will not propagate any new signals. The inhibition and directional control of propagation is needed to keep the system from over-stimulating itself. Early implementations simply used up all the resources of the hardware only moments after the initial stimulation.

The cinematic montage is made up of four layers allowing four images to be visible at a time. As the free-association signal propagates through the network, the units' IDs $^{31}$ and degrees of activation are fed into the montage mechanism. The mechanism is made up of four FIFOs - each corresponding to one of the layers. Activations are stored in the FIFOs in the order of activation. Each FIFO is emptied one item at a time. For each item popped, the system retrieves the corresponding image, fades it in, delays for a duration, and then fades it out. The duration the image is visible is proportional to the strength of the signal. The stronger the unit activation, the higher the value and the longer the duration the image is visible. The duration is calculated according to duration $(s)=1000 s+500$ where $s$ is the signal strength and the resulting duration is in milliseconds. The opacity of the image is also proportional to its signal strength where the stronger the activation, the more opaque the associated image is ( opacity $(s)=.8 s)$. The result is a montage of cascading sensor impressionsstarting with similar and brighter images that are visible for a longer duration. As the activation decays, dissimilar impressions become darker and are visible for shorter periods.

The mechanism behind this process is inspired by the work of Liane Gabora described in " $[t]$ he Cognitive Mechanisms Underlying the Creative Process" (Gabora, 2002). My interpretation of Gabora's theory considers creativity as a controlled form of free-association. The cascade of activations resemble how free-association could 
work in the human mind. In Gabora's theory, the network of memories is different in three ways when compared to the "Memory Association Machine" free-association system. Firstly, Gabora's theory considers memory as sparse, whereas the SOM organizes content into an organized spacial grid where all units are associated with some input during training - therefore containing no spaces. The folds, boundaries where nearby units are associated with very different input patterns, that some feature maps contain could be considered spaces. These folds can be seen by comparing the code-book vectors of adjacent units using the U-Matrix method (Ultsch and Siemon, 1989; Ultsch, 1993; Kraaijveld, 1992). At the time of writing the ann_som external only provides rudimentary feedback on the state of the codebooks. Secondly, "Memory Association Machine" stores entire images, whereas Gabora's theory considers each memory unit as micro-features of stimulus. Thirdly, Gabora's theory refers to a "controlled" association, where the free-association in "Memory Association Machine" is an emergent result of the interactions between the system's components and context.

\section{MACHINE CREATIVITY}

Boden (2004) defines creativity as "...the ability to come up with ideas or artifacts that are new, surprising and valuable". In the research domain surrounding "Memory Association Machine," the aspect of newness is the focus above surprise and value. As "Memory Association Machine" is meant to structure itself based on its embodied negotiation with its environment, newness comes from its ability to be different for each new context. The results of its embodied negotiation create a unique reflection on its context. Additionally, the system shifts over time as its context shifts. The diversity and complexity of the real-world environment should guarantee that the system never receives an identical stimulus twice. The value of the project is not in the machine's creative act (the systems external properties), but in the process that makes it possible. Boden specifies three classes of creativity:

- Combinational creativity is linking together known ideas that are not already associated.

- Exploratory creativity is accomplished by moving through the space of possibilities.

- Transformational creativity is the alteration of the space of possibilities.

Combinational creativity is inevitable in a connectionist network that supports learning. This is because the shift of the units' code-books change the topology of the network-combining the inputs in various ways. Exploratory creativity is also present in these systems as, through the learning process, the network is exploring the space of input possibilities. In order for a connectionist network to exhibit transformational creativity, it would have to be able to change the space of possibilities. The current combination of a SOM and model of free-association used in "Memory Association Machine" allow exploratory creativity since the free-association traverses its memory. At the very least the memory, at a snapshot in time, serves as the space of possibilities from which it can choose to be creative. Since the space of possibilities in the memory system is a constantly shifting field of experience, "Memory Association Machine" also exhibits transformational creativity through its ability to add to, and remove from, its space of possibilities over time. Even an identical memory traversal (which is already unlikely to repeat itself) would select an entirely different set of images from experience. As the SOM is a $12 \times 12$ grid of experiences, it has a fixed space of possibilities at a particular moment in time. The use of "Adaptive Resonance Theory" (Carpenter and Grossberg, 1994), "Incremental Grid Growing" (Blackmore and Miikkulainen, 1993), or "Growing Cell Structures" (Fritzke, 1991) networks 
could allow the memory system to create a new category for a new stimulus-without effecting the categories of previous experience. The space of possibilities would increase over time as the system gains more experience. The field of memory itself would then grow in response to the embodied context, rather than continually refining its finite number of units.

Lets consider creativity as a two-step process. Some originator, the kernel of creativity, creates a "new" item. This item then goes through a process of evaluation that filters all but the most new, surprising, and valuable items. In the case of "Memory Association Machine," the originator for creativity is the embodied context of the machine. Boden largely concentrates on the evaluative aspects of creativity and spends little time on the originator. In "Memory Association Machine," there is no mechanism that serves the role of the evaluator for the system's external properties. That is not to say that "Memory Association Machine" should not be able to evaluate the results of its own creativity, but that the evaluation should not be specified in advance, but come about as a result of its embodied process.

Boden's argument can be summed up in one statement: a creation can only be considered "creative" if it has been successfully evaluated as such. ${ }^{32}$ Of course, these two steps are both required for a creative result. Emphasizing one over the other is to only create a partial model of creativity. A more significant error would be to reduce creativity to evaluation since, without the originator, the mechanism has nothing to evaluate. The result of the first step in isolation may not originate something highly surprising or valuable, but certainly could originate something new. If we were to execute only the second step, evaluation, then nothing would originate at all. The hierarchy is clear-creativity is most dependent on the originator, and less on the evaluator as long as "newness" is the most important aspect of creativity.
As a counter to most of the literature in the area, I aim to put more focus on the seed of creativity, as apposed to its evaluation. From its initial conception, the purpose of "Memory Association Machine" is to originate - not to evaluate. The creation of a machine that originates, without being dependent on the artist, nor being random, is the foundation of this research project. The longterm challenge is the creation of potential systems where the seed of creativity is not dependent on randomness but only on the embodied process. This aspect of the research is connected with artificial life research, which is tied to abiogenisis. ${ }^{33}$ The originator $\rightarrow$ evaluation problem is analogous to a central concern of abiogenisis. Was it random fluctuations of early organic molecules, some form of self-organization, natural selection, or a process as of yet undiscovered that made life initially possible? The theories in abiogenisis are a potential source of technical and philosophical ideas important to the creation of artworks that relate to their context and are not predetermined in their external properties.

\section{Machines that are Intended to be Creative}

This section is a survey of selected artistic projects that, in my consideration, exhibit creative behaviour. The choice of projects is not meant to be exhaustive but to highlight a diverse set of applications of machine creativity. These projects involve both connectionist and non-connectionist approaches.

One of the most notable examples of "creative" machinery are the AARON programs written by Harold Cohen (Cohen, 1979, 1995) starting in 1973 and continuing to the present. As a collection of programs, AARON can "create" in a number of different painting styles. Each style uses a different variant of AARON that implements a different set of compositional rules. Some examples of these variants are: "abstract AARON," which creates abstract landscapes; 
"acrobat AARON," which creates acrobatic figures; and "jungle AARON," which creates scenes of figures in a complex jungle ground that evoke Gauguin. AARON programs contain sets of rules that encode specific compositional and stylistic laws that are specified by Cohen. Each element of the paintings - figures, grounds, and objects - are each a representation of the model those rules encode. The choices AARON makes are generated by a weighted random number generator and constrained by rules (Cohen, 1979). The results of these choices are applied to create paintings that, in a recent version, are physically produced by a painting machine. AARON has no sensory system. It receives no feedback from the results of its actions on the canvas. The system contains an internal representation that effects the placement, pose, and arrangement of items in the picture plane. AARON is the ultimate example of modernist creation. The internal model ${ }^{34}$ is realized in that perfect theoretical vision - regardless of the properties of the physical artifact. The only feedback between the physical artifact and AARON is through Cohen himself.

AARON could only be considered creative in a symbiotic relationship with its creator. ${ }^{35}$ Early "paintings" were drawn by AARON but painted by Cohen.${ }^{36}$ Cohen does not consider AARON an artist. AARON's artwork is the result of a collaboration between Cohen and AARON. Cohen believes that this software system is a natural approach to art-making because artistic composition is rule-based. While I can agree that graphic composition, in a particular style of painting, can be considered rule-based, it does not follow that all aspects of artistic creation are. The AARON software exhibits combinational and exploratory creativity, but not transformational creativity. It is unable to compose any choice that has not already been defined in rules specified by Cohen.

In 1981 David Cope started writing "Experiments in Musical Intelligence"(EMI) (Cope, 1996) in order to deal with a creative block in his own composition. The project started as an effort to automate the compositional process using the style of Cope's own compositions to date. The software uses a variation of Augmented Transition Networks (ATNs) (Woods, 1970), which were created to model the syntax of natural languages. This is the basis of a system that models the structure of musical compositions and creates "signatures" from the common aspects of multiple compositions. The elements of these signatures are then combined, in a second process, to form a new work that exhibits the style of the source composer. Clearly using combinational creativity, the software recombines the structures it sees in source-work. Since the space of possibility is limited to the "signature", created from input data, the system is unable to perform exploratory or transformational creativity. The system is fed abstractions of compositions as source material and is unable to perceive, let alone evaluate, the results of its processes.

David Rokeby has created two works that can be considered creative. ${ }^{37}$ The first exhibition of "The Giver of Names" (Rokeby, 1990) was in 1998 in Guelph, Canada. ${ }^{38}$ The system perceives the outside world through a video camera pointed at a pedestal. The floor around the pedestal is scattered with children's toys that the audience is free to place in the camera's view. "The Giver of Names" attempts to give names to the objects it sees. Associating their colour and shape with concepts in its knowledge-base, the system creates a free writing passage, written in proper grammatical structure, inspired by those objects. WordNet was used as the basis of the knowledge-base and expanded using various methods-including information returned from a "reading" system which extracts the relations between words from texts. This disembodied knowledge is then linked to the machine's sensory experience of the physical world. The result is grammatical sentences, whose grammatical rules are specified by the code, inspired by the machine's sensory experience. The choice of where to begin within the knowledge-base is not a result of agency in 
the system, but a response to the agency of the viewers' action.

"n-cha(n)t" (Rokeby, 2001) was first installed in 2001 in Banff, Canada and builds on some of the ideas of language and interpretation that are embodied in "The Giver of Names". "n-cha(n) $\mathrm{t}$ " is a cluster of independent systems that are connected in a network. Each of the system units is able to both hear and speak by accessing a knowledge-base analogous to the one used in "The Giver of Names". The hearing process attempts to interpret sound from a microphone and translate it into text. That translated text then stimulates the knowledge-base, which is shared between units, resulting in a free writing passage. The passage is then spoken by voice synthesis. The hearing apparatus is a highly directional microphone that picks up sounds only in close proximity and ignores the sounds from the unit's neighbours. The units communicate their "object of interest" 39 over an Ethernet network. In the absence of external stimuli, the units tend to chant a mix of phrases and sentences in synchrony. As all units in the network share the same knowledge-base, they tend to chant when they are not disturbed. The synchrony is an emergent result of the constancy of the perceived environment and the relational database across units. The chant can be disturbed by external stimulus. When the microphone of one unit picks up a sound, the result is a shift of its trajectory in the relational database. The chant can also be disturbed when the timing between units slips. ${ }^{40}$ Once all units are in synchrony, they chant the same sequence of words in unison. After the system has been disturbed it will eventually converge-where all units chant in unison.

Both "Giver of Names" and "n-cha(n)t" are embodied systems, as they are attached to the physical world through sensors that allow them to respond to their context. Their knowledge in the form of the relational database, on the other hand, is implanted into the system by the artist. It is not constructed through the system's experience. These systems show combinational creativity by pulling words from their knowledge-base to create texts. The associations in the knowledgebase change in response to sensory experience. The change remains in the system for a short period and dissipates over time. It is unclear if Boden would consider these systems capable of exploratory creativity. Although the vocabulary of these systems is fixed, the associations between those words are in flux. The space of words that follow is transformed in terms of both the words that preceded it, and the sensory experience of the machine. The space of possibilities is fixed at one moment, but the associations can change. The result is a space that changes from one moment to the next. It does not appear possible that the system could use a grammatical structure that is not encoded in the system.

George Legrady's "Pockets Full of Memories" (Legrady and Honkela, 2002) was made possible by a commission from the Centre Pompidou Museum of Modern Art in 2001. The project was revisited in 2003 and exhibited in the Dutch Electronic Arts Festival in Rotterdam, Netherlands. "Pockets Full of Memories" is one of the few artistic projects that makes use of a connectionist network. The system uses an implementation of the Kohonen SOM to organize content provided by the audience. The installation consists of a large projection and a number of kiosks with flat-bed scanners. The audience is encouraged to scan an image of some artifact in their possession. The kiosk then prompts the participant to answer questions about the meaning of the artifact. The answers to those questions are stored in a database and bound, as meta-data, to the images from the scanner. This meta-data is fed into the SOM and the categories of the images are visualized in the projection. Artifacts attached to similar attributes are plotted closer together than artifacts with dissimilar attributes. The SOM used in this project is a collaboration between George Legrady and Dr. Timo Honkela, who conducts research into artificial systems to study cognitive processes. 


\section{CONCLUSION \& FUTURE WORK}

At the time of writing, "Memory Association Machine" is running in a long-term installation at Simon Fraser University, where it is the platform of development for the author's M.Sc. thesis work. This stage of development concludes the first phase of intuitive development. The next stage will be to reflect on the behaviour of the system, and use that knowledge go back through the software to reconsider the arbitrary and intuitive choices.

One of the arbitrary choices that has a significant effect on the system is the choice of learning and neighbourhood functions. These functions were selected in the initial intuitive phase. The ann som implementation will be altered to include a function to set all the weights in the network to random values. Currently, the only way to randomize the weights, using ann_som, is to feed the object with a random value for each sensor. This is highly impractical in the case of "Memory Association Machine," where approximately four million random values need to be set.

The major remaining random variables include the direction in which the camera looks and the direction, and timing, of the propagation of freeassociation signals. A simple stimulus-response model of attention could be used to drive the direction in which the camera looks. This could be initiated by ambient sounds in the environment, which could inspire the attention of the camera. The long-term goal is that the direction in which the camera looks is a result of the free-association itself. The mechanism that feeds the system new stimulus would be driven by previous stimulus. It is not clear how this could be accomplished at this time. In a future version, an ideal mechanism to control the propagation of the free-association signal would be to degrade the activation signal by an amount proportional to the input space distance between the code-books of source and destination units. The result would be that free-associations within clusters of memories would last longer and tend to terminate once they near the folds in the
SOM. A reinforcement model could allow folds to be traversed, as some units would increase, rather than degrade, the activation signal. With this constraint in place, the activation could be set to propagate in all directions.

This project is an attempt to weave a meta-practise binding technique from AI with site-specific and responsive electronic media art practises. The meta-practise is grounded in the phenomenology of Merleau-Ponty and is developed through the production of artworks (embodied artifacts-asprocesses) that are meant to find their own relation to their context. The material of these creative machines is a fusion of the software (written by the artist) and the behaviour of the system (in collaboration with the artist).

\section{REFERENCES}

Agre, P. (1997). Computation and Human Experience. Cambridge University Press, ISBN 0521386039 .

Barker, E., Webb, N., \& Woods, K. (1999). The Changing Status of the Artist. Yale University Press. ISBN 0300077424.

Barr, A., \& Feigenbaum, E., (Eds.) (1981). The Handbook of Artificial Intelligence, 1. Morgan Kaufmann.

Blackmore, J., \& Miikkulainen, R. (1993). Incremental grid growing: encoding high-dimensional structure into a two-dimensional feature map. In Neural Networks, IEEE International Conference on, 1, 450-455).

Boden, M. A. (2004). The Creative Mind: Myths and Mechanisms. Routledge; London, 2nd edition.

Bogart, B. (2008). Memory association machine: An account of the realization and interpretation of an autonomous responsive site-specific artwork. Master's thesis, Simon Fraser University. 
Brooks, R. A. (1992). Intelligence without representation. Foundations of Artificial Intelligence, 47, 139-159.

Carpenter, G.A., \& Grossberg, S. .(1994). Adaptive Resonance Theory. Boston University, Center for Adaptive Systems and Dept. of Cognitive and Neural Systems.

Cohen, H. (1995). The further exploits of aaron, painter. Stanford Humanities Review, 4, 141158.

Cohen, H. (1979). What is an image? In Proceedings of IJCAI.

CollabNet. (2000). Subversion. http://subversion. tigris.org.

Cope, D. (1996). Experiments in musical intelligence. AR Editions.

Danks, M., Geiger, G., Zmölnig, J.M., Clepper, C., \& Tittle, J. II. (1995). Graphics environment for multimedia, http://gem.iem.at/.

Fritzke, B. (1991). Unsupervised clustering with growing cell structures. In Neural Networks. IJCNN-91-Seattle International Joint Conference on, 2, 531-536.

Gabora, L. M. (2002). Cognitive mechanisms underlying the creative process. In T. Hewett \& T. Kavanagh, (Eds.), Proceedings of the Fourth International Conference on Creativity and Cognition, (pp. 126-133).

Kaye, N. (2000). Site-specific Art: Performance, Place and Documentation. Routledge,. ISBN 0415185599 .

Kohonen, T. (2001). Self-Organizing Maps. Springer. ISBN 3540679219.

Kraaijveld, M.A. (1992). A non-linear projection method based on kohonen's topology preserving maps. Pattern Recognition, II. Conference B: Pattern Recognition Methodology and Systems, Proceedings, 11th IAPR Interna- tional Conference on, (pp. 41-45), Aug-3 Sep. doi: rm10.1109/ICPR.1992.201718.

Kwon, M. (2004). One Place After Another: SiteSpecific Art and Locational Identity. MIT Press. ISBN 026261202X.

Legrady, G., \& Honkela, T. (2002). Pockets full of memories: an interactive museum installation. Visual Communication, 1(2), 163-169. http://vcj. sagepub.com/cgi/content/abstract/1/2/163.

Medler,D.A.(1998). A brief history of connectionism. Neural Computing Surveys, 1, 61-101.

Merleau-Ponty, M. (1969). The Visible and the Invisible, trans. Evanston: Northwestern University Press.

Possiant, L. (2007). Media Art Histories, (pp. 229-250). MIT Press.

Puckette, M. (1996). Pure data. http://puredata. info.

R Development Core Team. (2007). R: A Language and Environment for Statistical Computing. $R$ Foundation for Statistical Computing, Vienna, Austria. http://www.R-project.org. ISBN 3-900051-070.

Rokeby, D. (1990). The Giver of Names. http:// homepage.mac.com/davidrokeby/gon.html.

Rokeby, D.. (2001). n-cha(n)t. http://homepage. mac.com/davidrokeby/nchant.html.

Turing, A. (2004). The Essential Turing: Seminal Writings in Computing, Logic, Philosophy, Artificial Intelligence, and Artificial Life, Plus the Secrets of Enigma, chapter Computing Machinery and Intelligence (1950). Oxford University Press, USA.

Ultsch, A. (1993). Self-organizing neural networks for visualization and classification. Information and Classification, (pp. 307-313).

Ultsch, A., \& Siemon, H. (1989). Exploratory data analysis: Using kohonen's topologypreserv- 
ing maps. Technical Report 329, University of Dortmund, Germany.

Wilson, S. (2002). Information Arts: Intersections of Art, Science and Technology. MIT Press.

Wilson, S. (1995). Artificial intelligence research as art. Stanford Electronic Humanities Review, 4(2).

Woods, W.A. (1970) Transition network grammars for natural language analysis. Commun. ACM, 13, 591-606, 1970.

Zmölnig, J.M. (2001). ann_som: Component of the Artificial Neural Network library for Pure Data. http://puredata.info/Members/dmorelli/ ann/?searchterm=neural.

\section{KEY TERMS}

Artificial Intelligence (AI): A discipline of computer science that seeks to create systems that can exhibit abilities similar to those of human beings (e.g. problem solving).

Connectionism: A thread of AI that is characterized by the development of systems, which are composed of numerous simple units that are massively interconnected and inspired by neurons.

FIFO (First in First Out): A type of buffer or stack where items can be removed in the same order in which they were added.

Self-Organizing Map (SOM): Also known as a Kohonen Network: an unsupervised artificial neural network designed to be an arbitrary pattern classifier.

Site-Specific Art: An art-form where the artwork is installed in a particular context and is meant to impact the viewer's reading of the work.

\section{ENDNOTES}

1 The phenomenological assumption is that the physical world is shared between subjects.

2 The "frame" is not referring to frame analysis, but a boundary that changes the meaning of what it contains simply through the act of isolating it from context.

3 Past frames are not just referring to those in this particular project, but to frames in all previous creative projects conducted by this artist.

4 A single artifact may also contain a number of components at the same level not depicted in this figure.

5 Action is considered the system's behaviour that results in the change of its external properties.

6 Possiant discusses the shift from artifact to interface. An interface, by definition, implies a process. Her argument then additionally supports a shift from artifact to process.

7 The intuitive approach involves the use of tacit knowledge in order to get a component functioning in the embodied system as quickly as possible.

8 Note that Wilson's characterization of creators as "artifact makers" indicates that even in the technological arts the object is often the central focus, rather than the process the artifact implements.

9 Robotics approaches to AI are also sources for potential technique.

10 VESA Display Power Management Signalling

11 "Topology" can be considered spacial structure or geometry.

12 The number of categories and dimensions are specified before training proceeds.

13 Units are also known as "nodes" and "neurons."

14 This measure is based on a direct pairwise comparison between the corresponding 
components of the input patterns. The measure of similarity is then unable to see two inputs, with identical blocks of values occupying different dimensions, as similar.

These values include the time images are kept during the dreaming and suspended states.

22 This is somewhat rare and always limited to a regional cluster-due to the SOM. ranges from 1 to 0 (inclusive) is that the code-books of few units are updated for each robust the SOM is - even when used with arbitrary initial conditions. Training can be accelerated by doing some pre-processing on the input patterns to determine initial codebooks. See Kohonen (2001) Section 3.7. At the time of writing ann_som(Zmölnig, 2001) does not implement an internal function to set code-books to random values. implementation, ifthecode-booksofallunits are equal, the unit with the largest index is always chosen as the initial BMU. of sensors and the size of the network. 2001) is the SOM implementation used. though it is fixed at 1 (opaque in GEM colour units) for all pixels in all frames. This is due to the high CPU usage of extracting the alpha values from the 30,000 element message in Pure Data. A possible solution would be a custom external to remove every $4^{\text {th }}$ element in a message.

The result of a neighbourhood size that iteration. The initial choice of this range was based on the assumption that ann_som was

normalizing the neighbourhood to the size of the network, which has proven not to be the case.

In the case of MAM, which trains over hundreds of thousands of iterations, the initial code-books are of little consequence.

25 Large changes result from a large neighbourhood and learning rate - small changes from a small neighbourhood and learning rate. For more information on the quality of the feature maps produced by MAM see Bogart (2008).

27 The traversal depends on the state of organization of the SOM.

28 Abstractions in Pure Data are roughly analogous to functions in procedural languages.

29 These are the neighbours of the free-association units, which are totally independent of the SOM neighbourhoods.

30 The signal degradation decreases to $10 \%$ during the sleeping state. This is to encourage longer free-associations in that state.

31 The ID in this case is the unique index, not a pair of $(x, y)$ indices.

32 For Boden, the creativity is the result of the two-step process, so before the evaluation the "newness" should not be considered creative at all, but simply as an unclassified response.

33 The study of the origin of life.

34 AARON's rules could be considered a model of creative intention.

35 Every "creative" machine depends on its creator to be creative.

36 Cohen has been known to paint a drawing differently than specified by AARON.

37 The characterization of these systems as creative is the interpretation of the author. The artist's intention was not centrally the construction of creative machines.

38 A prototype of "The Giver of Names" (Rokeby, 1990) was also exhibited in Toronto, Canada, in 1997. 
39 The "object of interest" is the concept in the knowledge-base that is currently being stimulated.
"Slips" occur because the timing mechanism of each unit is intentionally not synced to its neighbours, which results in some temporal drift. 